\title{
THE ARDUOUS JOURNEY TO BLACK HOLE FORMATION IN POTENTIAL GAMMA-RAY BURST PROGENITORS
}

\author{
Luc Dessart $^{1,2}$, Evan O'Connor $^{2}$, and Christian D. OtT ${ }^{2,3,4}$ \\ ${ }^{1}$ Laboratoire d'Astrophysique de Marseille, Université Aix-Marseille \& CNRS, UMR7326, \\ 38 rue Frédéric Joliot-Curie, 13388 Marseille, France; Luc.Dessart@ oamp.fr \\ 2 TAPIR, Mailcode 350-17, California Institute of Technology, Pasadena, CA 91125, USA; evanoc@tapir.caltech.edu, cott@ tapir.caltech.edu \\ ${ }^{3}$ Institute for the Physics and Mathematics of the Universe (IPMU), The University of Tokyo, Kashiwa, Japan \\ Received 2012 March 9; accepted 2012 May 18; published 2012 July 6
}

\begin{abstract}
We present a quantitative study on the properties at death of fast-rotating massive stars evolved at lowmetallicity-objects that are proposed as likely progenitors of long-duration $\gamma$-ray bursts (LGRBs). We perform one-dimensional+rotation stellar-collapse simulations on the progenitor models of Woosley and Heger, and critically assess their potential for the formation of a black hole and a Keplerian disk (namely, a collapsar) or a proto-magnetar. We note that theoretical uncertainties in the treatment of magnetic fields and the approximate handling of rotation compromise the accuracy of stellar-evolution models. We find that only the fastest rotating progenitors achieve sufficient compactness for black hole formation while the bulk of models possess a core density structure typical of garden-variety core-collapse supernova $(\mathrm{SN})$ progenitors evolved without rotation and at solar metallicity. Of the models that do have sufficient compactness for black hole formation, most of them also retain a large amount of angular momentum in the core, making them prone to a magneto-rotational explosion, therefore preferentially leaving behind a proto-magnetar. A large progenitor angular-momentum budget is often the sole criterion invoked in the community today to assess the suitability for producing a collapsar. This simplification ignores equally important considerations such as the core compactness, which conditions black hole formation, the core angular momentum, which may foster a magneto-rotational explosion preventing black hole formation, or the metallicity and the residual envelope mass which must be compatible with inferences from observed LGRB/SNe. Our study suggests that black hole formation is non-trivial, that there is room for accommodating both collapsars and proto-magnetars as LGRB progenitors, although proto-magnetars seem much more easily produced by current stellar-evolutionary models.
\end{abstract}

Key words: gamma-ray burst: general - hydrodynamics - stars: evolution - stars: mass-loss - stars: rotation supernovae: general

Online-only material: color figures

\section{INTRODUCTION/CONTEXT}

All stars with masses initially between $\sim 8$ and $\sim 150 M_{\odot}$ eventually form a degenerate core that inevitably collapses to form a proto-neutron star. Much less certain is its subsequent evolution, the potential formation of a black hole, and the powering of a supernova ( $\mathrm{SN}$ ) explosion, sometimes associated with a long-duration $\gamma$-ray burst (LGRB). The situation is deceptively simple and the outcome rests fundamentally on the solution to an energy problem. An explosion or a fizzle depends on the efficiency with which the system can extract the prodigious gravitational energy released during collapse. There are two principal forms of energy at disposal. The first one is the gravitational-binding energy liberated by the collapsing star and in particular its degenerate core. The second is its rotational energy (actually drawn from gravitational energy), which is a function of the angular-momentum distribution and budget in the progenitor star. Understanding how these two energy sources can be channeled to power relativistic and non-relativistic ejecta in core-collapse $\mathrm{SNe}$ and leave behind a neutron star, a fastrotating pulsar, a magnetar, or a black hole, has been the subject of numerous studies and the source of much debate (Bethe 1990; Woosley 1993; Herant et al. 1994; Burrows et al. 1995, 2006; Janka \& Müller 1996; Wheeler et al. 2000; Kitaura et al. 2006; Buras et al. 2006b; Murphy \& Burrows 2008; Nordhaus et al.

\footnotetext{
4 Alfred P. Sloan Research Fellow.
}

2010; Pejcha \& Thompson 2012; Hanke et al. 2011; Takiwaki \& Kotake 2011).

Thermal $\mathrm{MeV}$ neutrinos are abundantly radiated from the optically thick, dense, and hot proto-neutron star, allowing its internal energy to be released on a diffusion timescale. In the neutrino mechanism for core-collapse SN explosions (Bethe \& Wilson 1985), the absorption in the infalling mantle of $\sim 10 \%$ of this neutrino flux may alone lead to the revival of the stalled shock and the ejection of the progenitor envelope with an asymptotic kinetic energy of up to $1 \mathrm{~B}\left(10^{51} \mathrm{erg}\right.$; Kitaura et al. 2006; Buras et al. 2006a, 2006b). But this generic mechanism should not, however, be the origin of the larger explosion energies of $\sim 10 \mathrm{~B}$ inferred for a small fraction of core-collapse SNe. Instead, their scarcity calls for exceptional circumstances, which seem intricately related to fast progenitorcore rotation (Burrows et al. 2007; Takiwaki \& Kotake 2011). It is probable that most stars contain some angular momentum at the time of death, either because they did not lose it completely through the combined effects of magnetic torques and stellarwind mass loss (Maeder \& Meynet 2000; Meynet \& Maeder 2000, 2005; Heger et al. 2000, 2005; Hirschi et al. 2004, 2005), or because they gained it from a companion star in a binary system (Wellstein \& Langer 1999; Petrovic et al. 2005; Cantiello et al. 2007). As the envelope collapses, the rotational energy increases. During this process, the inner core $\left(\lesssim 0.5 M_{\odot}\right)$ spins up by about three orders of magnitude and remains in solid-body rotation, while the outer core develops 
a differentially rotating profile (Ott et al. 2006). The energy associated with rotation can be large $(\mathcal{O}(10 \mathrm{~B}))$ and tapped by instabilities developing at the surface of the proto-neutron star, in particular the magneto-rotational instability (MRI; Balbus \& Hawley 1991; Akiyama et al. 2003; Obergaulinger et al. 2009). Numerical simulations for fast-rotating progenitor stars suggest that the magneto-rotational mechanism of explosion is promising and offers a very attractive explanation for the existence of highly energetic SNe (LeBlanc \& Wilson 1970; Bisnovatyi-Kogan et al. 1976; Wheeler et al. 2000; Yamada \& Sawai 2004; Moiseenko et al. 2006; Burrows et al. 2007; Dessart et al. 2008; Takiwaki \& Kotake 2011). However, this mechanism relies fundamentally on the assumption that the MRI can increase the magnetic field to the required values - an assumption that has not yet been shown numerically in the full core-collapse context, although preliminary investigations in this sector are promising (Obergaulinger et al. 2009). Hence, combined with the diversity of progenitor-core properties, these two mechanisms alone, the neutrino and the magneto-rotational mechanism, may explain the diversity of core-collapse $\mathrm{SNe}$, potentially encompassing two orders of magnitude in explosion energy, from the low-luminosity SNe II-Plateau (Pastorello et al. 2004) to highly energetic SNe Ic (Mazzali et al. 2002).

A great puzzle is then to understand the necessary departures from this general core-collapse scenario to produce an LGRB in addition to an SN explosion, as spectroscopically confirmed in, to date, six LGRB/SN pairs (for a recent compilation, see Berger et al. 2011). This requires that $\sim 0.1-1 \mathrm{~B}$ be injected into a low-mass, baryon-deficient collimated region (at the origin of $\gamma$-rays) and that at the same time $\sim 10 \mathrm{~B}$ be injected quasi-isotropically to eject the progenitor envelope (at the origin of the $\mathrm{SN}$ thermal emission observed in the optical). The very low occurrence rate of LGRB/SN per core-collapse SN of $\lesssim 1 \%$ (Guetta \& Della Valle 2007) calls for progenitor properties that are rarely encountered in star formation/evolution. Interestingly, an unambiguous diversity emerges among LGRB/SN observations, necessarily translating into a significant range for the inferred properties of the SN ejecta, with proposed masses and kinetic energies possibly varying by a factor of 5-10 for both (Berger et al. 2011). Unfortunately, a significant uncertainty is associated with such inferences. For example, Iwamoto et al. (1998) propose an ejecta mass of $11 M_{\odot}$ with a total energy of 20-50 B for GRB980425/ SN1998bw, but Woosley et al. (1999) reproduce the light curve with an ejecta of $\sim 5 M_{\odot}$ and a total energy of $22 \mathrm{~B}$. Such differences are not surprising since both spectra and light curves must be modeled simultaneously and with allowance for the complicated non-LTE non-thermal and time-dependent effects controlling the radiative transfer. The exceptionally fast ejecta expansion of hypernovae is expected to strengthen the timedependent effects seen in "standard" core-collapse SNe (Dessart $\&$ Hillier 2008) while the large production of ${ }^{56} \mathrm{Ni}$ and significant mixing may sizably affect line-profile shapes from which the expansion rate is inferred (Dessart et al. 2012).

Two LGRB central-engine models are currently favored. They suggest that the key components for a successful LGRB/SN are a compact progenitor with a short light-crossing time of $\sim 1 \mathrm{~s}$ and fast rotation at the time of collapse. One is the collapsar model (Woosley 1993; MacFadyen \& Woosley 1999; MacFadyen et al. 2001): a fast-rotating progenitor fails to explode in its early post-bounce phase and instead forms a black hole, while the infalling envelope eventually forms a Keplerian disk feeding the hole on an accretion/viscous timescale comparable to that of the LGRB. It is within this disk that the SN explosion is triggered and the ${ }^{56} \mathrm{Ni}$ synthesized. The other model involves a proto-magnetar (Wheeler et al. 2000; Bucciantini et al. 2008; Metzger 2010; Metzger et al. 2011) in which the LGRB is born after a successful SN explosion (either by the neutrino or the magneto-rotational mechanism, although the latter seems more likely given the rapid rotation required for the magnetar) and the ejection of the overlying envelope (or at least the onset of the ejection of the inner envelope layers that clear the proto-neutron star surface). Fast rotation in the proto-neutron star permits the huge enhancement of the magnetic field energy and stresses, which strengthen as the proto-neutron star cools and contracts, eventually giving rise to relativistic ejecta $(\sim 10 \mathrm{~s}$ after the onset of collapse, once the neutrino-driven wind decays away). In both models, rotation is key to control the dynamics in a unique way. It is also key to allow the simultaneous ejection of baryondeficient material at relativistic speeds over a small solid angle and the quasi-spherical ejection of the progenitor envelope.

A critical difference between the collapsar model and the proto-magnetar model is that the collapsar has to form a black hole. Being so central to the model, it is legitimate to investigate what conditions black hole formation in this context (Woosley 2011). Surprisingly, little has been done on this problem. Numerous simulations so far have focused on the early collapse phase and the revival of the SN shock, stopping too early to make any statement concerning black hole formation. In one dimension (1D), several studies have investigated the neutrino signal and progenitor dependence of non-rotating failed $\mathrm{SNe}$ (Liebendörfer et al. 2004; Sumiyoshi et al. 2007; Fischer et al. 2009; O’Connor \& Ott 2011). Sekiguchi \& Shibata (2011) have performed two-dimensional (2D) simulations of black hole formation and the subsequent formation of an accretion disk, although with initial conditions that are incommensurate with currently suggested LGRB progenitors. In three dimensions, Ott et al. (2011) performed fully general-relativistic simulations of black hole formation, though using a simplified soft equation of state (EOS) that favored it. Finally, other simulations have started from a pre-existing black hole and investigated the powering of the jet at the origin of the LGRB (Aloy et al. 2000; Proga et al. 2003; Zhang et al. 2004; Lindner et al. 2010), or the longer-term synthesis of ${ }^{56} \mathrm{Ni}$ in this unusual context (Milosavljević et al. 2012). No simulation has ever demonstrated from first principles, and thus convincingly, the validity of the collapsar model, i.e., that the progenitors proposed for this model would indeed proceed through each and every necessary step: collapse, formation of a proto-neutron star, failure of the shock revival, formation of a black hole followed by that of a Keplerian disk, and finally the powering of both the LGRB and the SN, including the synthesis of a generally large amount of ${ }^{56} \mathrm{Ni}$ by core-collapse $\mathrm{SN}$ standards. This is an obvious shortcoming of all theoretical investigations on the collapsar model and its proposed progenitors. Similar gaps in the modeling of proto-magnetar-driven LGRBs exist: the early magneto-rotational core-collapse SN evolution has been modeled in 2D (Burrows et al. 2007; Takiwaki \& Kotake 2011) and so has the phase in which relativistic outflows are driven (Bucciantini et al. 2007, 2008, 2009; Komissarov \& Barkov 2007), but the evolution connecting the two phases has not been modeled. The robustness of the magneto-rotational explosion mechanism largely rests on the efficiency of angularmomentum transport, and in particular the extraction of the free energy stored (and replenished through accretion) in differential rotation at the surface of the proto-neutron star. The failure to 
extract this energy on short timescales may, however, facilitate black hole formation, although it may also compromise energy extraction in the collapsar model. These complicated issues require detailed modeling to build upon the promising results of Thompson et al. (2005) and Obergaulinger et al. (2009). To this day, the collapsar model has been studied more extensively than the proto-magnetar model for LGRBs, so the latter may look more promising in some ways in part because of the lesser scrutiny it has received.

In this paper, we focus on one important aspect of the collapsar model to validate, or invalidate, the assumption, often made but so far never checked, that the LGRB progenitor models available in the literature indeed collapse to form a black hole. We do this by performing hydrodynamical simulations of the LGRB progenitor models of Woosley \& Heger (2006) using the code GR1D (O'Connor \& Ott 2010). This issue is critical for testing the potential of progenitor stars for producing LGRBs via the collapsar mechanism, but may also serve to diagnose an attractive channel for the formation of protomagnetars. Such "failed" collapsars (because they explode before forming a black hole) represent a serious alternative for the production of LGRBs, although they have their own caveats (Metzger et al. 2011). Admittedly, the phenomenon of core collapse, bounce, and the events that follow are fundamentally multi-dimensional. We believe, however, that much can be learned from 1D simulations of the kind presented here. For example, the mass-accretion rate onto the proto-neutron star is largely determined by the angular-averaged density profile of the progenitor star, which we capture accurately. Our 1D exploration reveals the landscape of core properties at bounce and quantifies fundamental differences between progenitors. In the next section, we start by reviewing results from stellarevolution models for LGRB progenitors. We then describe our methodology for the GR1D simulations of LGRB progenitors available in the literature. We make a short digression in Section 4 to discuss the rotational properties of the collapsed cores of massive stars. In Section 5, we present our results before concluding in Section 6.

\section{STELLAR-EVOLUTION MODELS OF LGRB PROGENITORS}

Stellar-evolution calculations have been performed to investigate the mass, rotation, and metallicity requirements for producing fast-rotating pre-collapse stars. Both single- (Hirschi et al. 2005; Yoon \& Langer 2005; Woosley \& Heger 2006; Georgy et al. 2009) and binary-star (Petrovic et al. 2005; Cantiello et al. 2007 ) evolutionary scenarios have been investigated. Fast rotation of the proto-stellar core is clearly critical to procure a large angular momentum to the star initially. If the rotation rate attained is sufficiently large, the star may even evolve chemically homogeneously and avoid a supergiant phase, which is known to sap the core of its rotation through the effects of magnetic torques. Such fast-rotating chemically homogeneous stars also naturally die as H-deficient He-poor Wolf-Rayet (W-R) stars. Low metallicity quenches the stellar-wind mass-loss rate, a condition that may be more important for a single-star scenario than for the binary-star scenario (Yoon et al. 2006; Cantiello et al. 2007). While the general outcome of these simulations is that it is possible to produce massive stars with a rapidly spinning core/envelope at death, it is difficult to compare the final properties of published models. Indeed, models are rarely evolved all the way to an iron core. The treatments of mixing, mass loss, and angular-momentum loss/transport differ. Magnetic fields may or may not be included and when they are, the prescription may differ (Spruit 2002; Zahn et al. 2007).

Furthermore, all these studies remain speculative about the outcome of collapse for such progenitors. They argue for black hole formation and the formation of a disk based on order of magnitude estimates, rather than detailed numerical simulations. For a start, of all the LGRB progenitor simulations, only those of Woosley \& Heger (2006) are evolved all the way to the onset of collapse. In simulations halted well before, the iron-core mass is estimated from the CO-core mass (Hirschi et al. 2005) or is simply not considered in the discussion (Yoon \& Langer 2005). Most studies consider a model viable for producing a collapsar based exclusively on the angular-momentum budget of the inner $3 M_{\odot}$, and perform no checks on the likelihood of forming a black hole: its formation is deemed so obvious that the discussion of any alternate scenario is generally omitted.

Differing in their criteria and approaches for selecting collapsar candidates, some studies may yield progenitor-mass ranges that do not even overlap: using an angular-momentum criterion, Yoon \& Langer (2005), Woosley \& Heger (2006), and Yoon et al. (2006) favor progenitor stars with a main-sequence mass below $\sim 30 M_{\odot}$. In contrast, Hirschi et al. (2005), arguing for the need for both a large angular momentum, a large iron core, and a WO stellar type at death, favor progenitors with a mainsequence masses above $\sim 35 M_{\odot}$ (magnetic fields are not treated in this work, though).

Recent studies suggest that selecting collapsar progenitors based exclusively on a large angular-momentum budget may be too simplistic. For example, the magneto-rotational explosion invoked to explain hypernovae derives its energy from this same large core angular momentum (via the MRI and the strongly differentially rotating layers in the postshock region). This mechanism does not obviously accommodate the formation of a black hole, as demonstrated by Dessart et al. (2008). They simulated the collapse of the core and the development of a magneto-rotational explosion in model 35OC of Woosley \& Heger (2006) and found that rotational energy of order $10 \mathrm{~B}$ is readily available to launch an $\mathrm{SN}$ ejecta on a timescale of a few $100 \mathrm{~ms}$. They furthermore found that accretion is easily shut off by the developing explosion and that the proto-neutron star mass fails to grow to the instability threshold for black hole formation. However, the simulations of Dessart et al. (2008) did not resolve the MRI but instead used an equipartition ansatz to estimate the magnitude of the MRI-amplified magnetic fields. In reality, magnetic field reconnection may, for example, compromise the dynamical potential of magnetic stresses, channeling magnetic energy into heat to be radiated away by neutrinos. This and other alternatives have been studied by Thompson et al. (2005) under the general form of viscous dissipation. They find that the extra energy deposition can, in some cases, considerably alter the post-bounce dynamics and generate a vigorous explosion.

O'Connor \& Ott (2011) studied black hole formation based on a variety of progenitor models characterized by different main-sequence mass, metallicity, and rotation rate. They find that the outcome of collapse can be anticipated from the bounce compactness of the progenitor-core structure and in particular that of the region inside $2.5 M_{\odot}$, which corresponds approximately to the maximum mass that a proto-neutron star can have and remain in hydrostatic equilibrium. They find that higher-mass progenitors published in the literature do not always have larger iron cores and therefore that they are not necessarily more prone to black hole formation. They also reveal 
considerable diversity in progenitor-core structure, even for the same main-sequence mass. Some stellar-evolution studies obtain a monotonic increase of the iron-core mass (or bounce compactness; see Figure 9 of O'Connor \& Ott 2011) versus main-sequence mass (Limongi \& Chieffi 2006; see also Hirschi et al. 2004, 2005), while some show an anti-correlation beyond $\sim 40 M_{\odot}$ (Woosley \& Heger 2007). The primary reason for this is differing prescriptions for rate and time of mass loss, one of the major uncertainties in massive-star evolution (see also the discussions in Hirschi et al. 2005 and O'Connor \& Ott 2011).

\section{METHODS AND INITIAL MODEL SET}

In this work, we use the open-source, spherically symmetric, general relativistic, Eulerian hydrodynamics code GR1D (O'Connor \& Ott 2010). Rotation is included through a centrifugal-acceleration term in the momentum equation-this is the most important dynamical feature of rotation relevant to core collapse. However, GR1D cannot account for the associated deviations from spherical symmetry nor any angular-momentum redistribution. We select the EOS from Lattimer \& Swesty (1991) characterized by a nuclear incompressibility of $220 \mathrm{MeV}$ (hereafter referred to as the LS220 EOS). This EOS provides the best match to both mass and mass-radius constraints from observations and nuclear theory (Demorest et al. 2010; Özel et al. 2010; Steiner et al. 2010; Hebeler et al. 2010). GR1D uses an efficient neutrino leakage/heating scheme that qualitatively reproduces the salient features of neutrino transport. We refer the reader to O'Connor \& Ott $(2010,2011)$ for additional details on GR1D and our methodology.

As described above, the only stellar-evolutionary models for LGRB progenitors that are evolved until the onset of collapse are those proposed by Woosley \& Heger (2006). We thus focus on their model data set for our investigation on the dynamics of the core-collapse SN engine and the potential formation of a black hole in the collapsar context. Using KEPLER, Woosley \& Heger (2006) investigated a rather narrow range of progenitor masses, but varied the initial rotation rate (solid-body rotation is assumed initially) and environmental metallicity from solar to $1 \%$ solar (with an additional tunable factor as low as 0.1 for the metallicity-dependent mass-loss rate, equivalent to a reduction in metallicity by a factor of 100 in their mass-loss prescription). Arguing that the inferred mass of LGRB/SN ejecta known in 2006 is on the order of $10 M_{\odot}$, and since higher-mass stars may lose too much angular momentum through stellar winds (even at low metallicity), they focused primarily on lower-mass progenitors, with main-sequence masses of 12 and $16 M_{\odot},{ }^{5}$ with the exception of one $35 M_{\odot}$ model set.

We adopt the same nomenclature as for their 12,16, and $35 M_{\odot}$ models. It comprises the model's main-sequence mass, followed by a letter denoting the environmental metallicity ("S" for solar, "O" for $10 \%$ solar, and "T" for $1 \%$ solar). An additional letter is appended to individualize the models done with different $\mathrm{W}-\mathrm{R}$ mass-loss rate prescriptions, allowing or not for magnetic effects, and the total angular momentum of the star. $16 M_{\odot}$ helium models are denoted by "HE16" followed by an individualizing capital letter.

In this work, we simulate the collapse and post-bounce evolution with GR1D for all these progenitor models, with a primary focus on determining their ability to produce the key features of the collapsar model: a black hole together with a

\footnotetext{
5 They also perform simulations for $16 M_{\odot}$ helium cores and find comparable outcomes.
}

Keplerian disk. As we discuss in the following section, black hole formation is not obviously guaranteed in any of these dying stars.

\section{NOTES ON ROTATING CORE COLLAPSE}

Since LGRBs seem fundamentally related to rapid rotation, it is useful to summarize a few facts and concepts related to the gravitational collapse of rotating iron cores in massive stars. First, it is reasonable to assume (which is borne out by simulations, e.g., Heger et al. 2005) that the iron core, in its pre-collapse state, will be approximately uniformly rotating. Such a solid-body rotation corresponds to the lowest energy state at fixed total angular momentum and will be assumed on a secular timescale by any rotating fluid that has some means to redistribute angular momentum.

Rotating core collapse, even for the high pre-collapse rotation rates of some of the potential LGRB progenitors that we consider in this study, proceeds qualitatively in a very similar fashion to non-rotating collapse as long as the ratio of the centrifugal acceleration $a_{\text {cent }}$ to the gravitational acceleration $a_{\text {grav }}$, is small,

$$
\frac{a_{\text {cent }}}{a_{\text {grav }}}=\frac{\Omega^{2}(r) r}{G M(r) r^{-2}}=\frac{\Omega^{2}(r) r^{3}}{G M(r)} \ll 1 .
$$

Due to angular-momentum conservation, the angular velocity behaves as $\Omega(r) \propto r^{-2}$. M(r) stays constant for a collapsing mass shell, and, thus, the above ratio increases during collapse as $r^{-1}$ and may potentially become large for small radii.

In the case of $a_{\text {cent }} / a_{\text {grav }} \ll 1$, the collapsing rotating iron core will behave like a non-rotating core and separate into a subsonically collapsing inner core $\left(\left|v_{r}(r)\right|<c_{s}(r)\right)$ and a supersonically collapsing outer core $\left(\left|v_{r}(r)\right|>c_{s}(r)\right)$. The inner core exhibits a self-similar (homologous) velocity profile, $v(r) \propto r$, until core bounce and shock formation (Goldreich \& Weber 1980). After core bounce, the inner core material forms the core of the proto-neutron star and outer core material accumulates at its edge. The mass of the inner core at bounce is typically $\sim 0.5 M_{\odot}$ for non-rotating cores, set by the EOS of relativistic electrons, the trapped lepton number, core entropy, and gravity (Burrows \& Lattimer 1983). It increases monotonically, though slowly, with increasing pre-collapse rotation rate. For cores that reach $a_{\text {cent }} / a_{\text {grav }} \approx 1$, the mass of the inner core will be increased to $\gtrsim 0.7 M_{\odot}$ (Dimmelmeier et al. 2008).

Since the inner core is collapsing homologously, we can introduce a homology parameter $\alpha(t)$, so that

$$
r(t)=\alpha(t) r_{0}
$$

where $r(t)$ is the radius of a collapsing fluid element at time $t$ and $r_{0}$ is its initial radius. This must hold for any mass shell within the inner core. Two collapsing fluid elements located initially at $r_{1}$ and $r_{2}$, and rotating with a frequency $\Omega_{1}=j_{1} / r_{1}^{2}$ and $\Omega_{2}=j_{2} / r_{2}^{2}$ conserve angular momentum, $\Omega_{1}^{\prime} r_{1}^{\prime 2}=j_{1}^{\prime}=j_{1}=\Omega_{1} r_{1}^{2}$. Homology implies $r_{1}^{\prime}=\alpha(t) r_{1}$, and $r_{2}^{\prime}=\alpha(t) r_{2}$, therefore

$$
\frac{\Omega_{1}^{\prime}\left(r_{1}^{\prime}\right)}{\Omega_{2}^{\prime}\left(r_{2}^{\prime}\right)}=\frac{j_{1} / r_{1}^{\prime 2}}{j_{2} / r_{2}^{\prime 2}}=\frac{j_{1}}{j_{2}} \frac{\alpha(t)^{2} r_{2}^{2}}{\alpha(t)^{2} r_{1}^{2}}=\frac{\Omega_{1}}{\Omega_{2}} .
$$

Since this property holds for any mass shell within the inner core, the rotational profile must be preserved under homologous 


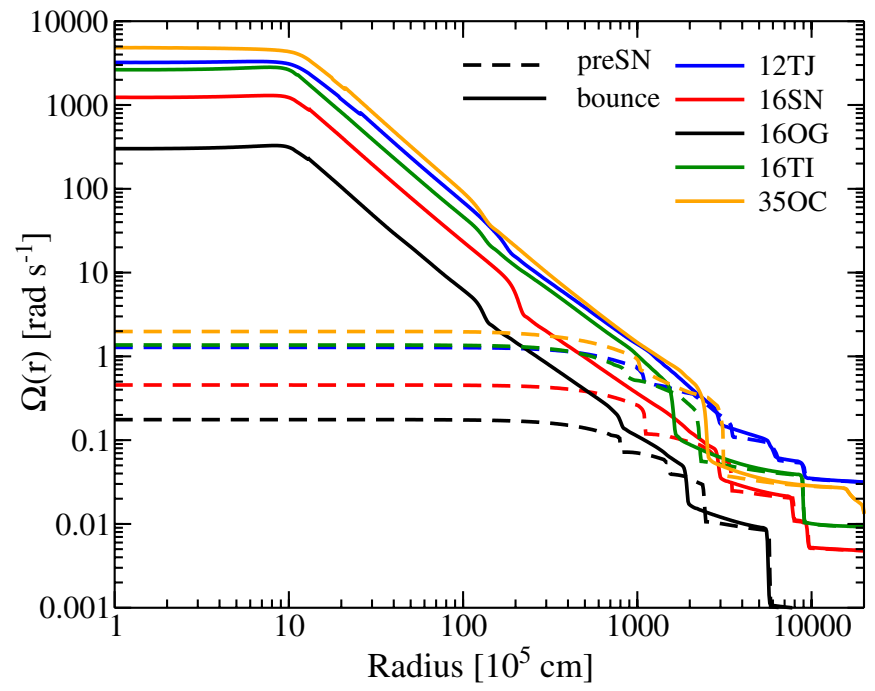

Figure 1. Angular velocity $\Omega(r)$ vs. radius $r$ at both the pre-SN stage (dashed lines) and at core bounce (solid lines) for selected models of Woosley \& Heger (2006). The inner homologously collapsing core maintains its initial uniform rotation throughout collapse.

(A color version of this figure is available in the online journal.)

collapse. In practice, because the pre-collapse cores of massive stars are always in solid-body rotation, so are the inner cores of the proto-neutron star at bounce. Outside of the homologously collapsing core, the self-similar relation of Equation (2) does not hold. Most generally, gradients in the rotation rate develop due to the underlying density gradients in the hot postshock region and in the supersonically infalling region ahead of the accretion shock.

Based on these arguments, we expect an early post-bounce rotational profile that is approximately uniform within the inner $0.5-0.7 M_{\odot}$ (out to $\sim 10-15 \mathrm{~km}$ in radius) and strongly differential at larger radii. This is confirmed by Figure 1 which shows $\Omega(r)$ at bounce as obtained with GR1D for a variety of models considered in this study. We also show the rotational profile at the onset of collapse. This result is not entirely new, but has previously been pointed out by Ott et al. (2006) in the context of $1 \mathrm{D}$ and 2D rotating core-collapse simulations.

Since uniform rotation is the lowest energy state, the shear energy of differential rotation is to be interpreted as a free energy that will be tapped by any process (e.g., nonaxisymmetric rotational shear instabilities, viscosity, or the MRI) capable of redistributing angular momentum. Viscosity would lead to additional heating in the postshock region to enhance the neutrino mechanism (Thompson et al. 2005) while the MRI action could strengthen the magnetic fields, driving bipolar outflows in the magneto-rotational mechanism (Burrows et al. 2007).

In our simulations, we estimate the available free energy of differential rotation by computing the difference in rotational energy of the proto-neutron star model in GR1D and the rotational energy of a uniformly spinning proto-neutron star of the same angular momentum and moment of inertia,

$$
F_{\text {rot }}=T-\frac{I \bar{\omega}^{2}}{2},
$$

where from O'Connor \& Ott (2010), for GR1D,

$$
T=\frac{4 \pi}{3} \int_{0}^{R_{\mathrm{PNS}}} \rho h X W^{2} v_{\varphi}^{2} r^{2} d r
$$

$$
\begin{gathered}
I=\frac{8 \pi}{3} \int_{0}^{R_{\mathrm{PNS}}} \rho h X W^{2} r^{4} d r \\
\bar{\omega}=\int_{0}^{R_{\mathrm{PNS}}} \rho h X W^{2} r v_{\varphi} r^{2} d r / \int_{0}^{R_{\mathrm{PNS}}} \rho h X W^{2} r^{4} d r
\end{gathered}
$$

where $T$ is the rotational energy, $I$ is the moment of inertia, and $\bar{\omega}$ is the uniform rotation frequency, $h$ is the specific enthalpy, $X^{2}$ is the $g_{r r}$ component of the metric, $W$ is the Lorentz factor, and $v_{\varphi}$ is the angular velocity. We take $R_{\mathrm{PNS}}$ to be the radius where the matter density, $\rho=10^{10} \mathrm{~g} \mathrm{~cm}^{-3}$.

\section{RESULTS}

We have performed simulations for the entire set of KEPLER models published in Woosley \& Heger (2006). ${ }^{6}$ We first consider the rapidly spinning progenitors evolved without magnetic fields. All these models have a dimensionless Kerr spin $\left(a^{\star}=\right.$ $J c / G M^{2}$ ) at $3 M_{\odot}$ greater than unity (with the exception of model HE16J, which has $a^{\star}=0.91$ ) and are thus considered as promising collapsar candidates by Woosley \& Heger (2006). Unfortunately, when evolved with GR1D, the collapsing iron core of all such models halts its collapse and expands - these models do not experience core bounce within a few seconds of evolution in GR1D. We associate this problem with the neglect of the centrifugal acceleration in the momentum equation in KEPLER, an approximation that fails in the fastest rotating models. This term is included in GR1D. The mismatch suggests that their fastest models may be significantly affected by the addition of this term. Even if they did collapse, it is not clear that such extremely fast rotating cores would avoid a centrifugal bounce.

In the remainder of this paper, we thus limit our discussion to models evolved with magnetic fields and therefore subject to magnetic torques during their evolution. Of the 46 models that fulfill this criterion, we identify four additional models (12OM, 16TJ, 35OD, and HE16G) that do not collapse but instead expand when restarted with GR1D. We exclude these as well from our study. Finally, for reference and completeness, we include the non-rotating models associated with each series (12SA, 12OA, 12TA, 16SA, 16OA, 16TA), making a total of 48 models. Each simulation is continued after core bounce until a black hole forms or until a time of $3.5 \mathrm{~s}$ has passed, whichever comes first. We present the results for these 48 models evolved with magnetic fields in Table 1 (for the table layout, we group models in bundles first of increasing mass, then of decreasing metallicity, and finally in alphabetical order, which generally corresponds to an increased initial rotation rate).

As advocated by O'Connor \& Ott (2011), the bounce compactness is a robust quantity for diagnosing the propensity to black hole formation, which is largely determined by the spatial extent encompassed by the $2.5 M_{\odot}$ Lagrangian mass coordinate at core bounce in the progenitor core. To avoid introducing biases associated with the non-uniform conditions in the progenitor simulations (KEPLER models are not all evolved to the same central density on their collapse trajectory), this compactness is unambiguously evaluated at the time of bounce. The formal definition of this core compactness is

$$
\xi_{M}=\left.\frac{M / M_{\odot}}{R\left(M_{\text {bary }}=M\right) / 1000 \mathrm{~km}}\right|_{t=t_{\text {bounce }}},
$$

\footnotetext{
6 Models are available from http://homepages.spa.umn.edu/ alex/GRB2/.
} 
Table 1

Progenitor Model Properties

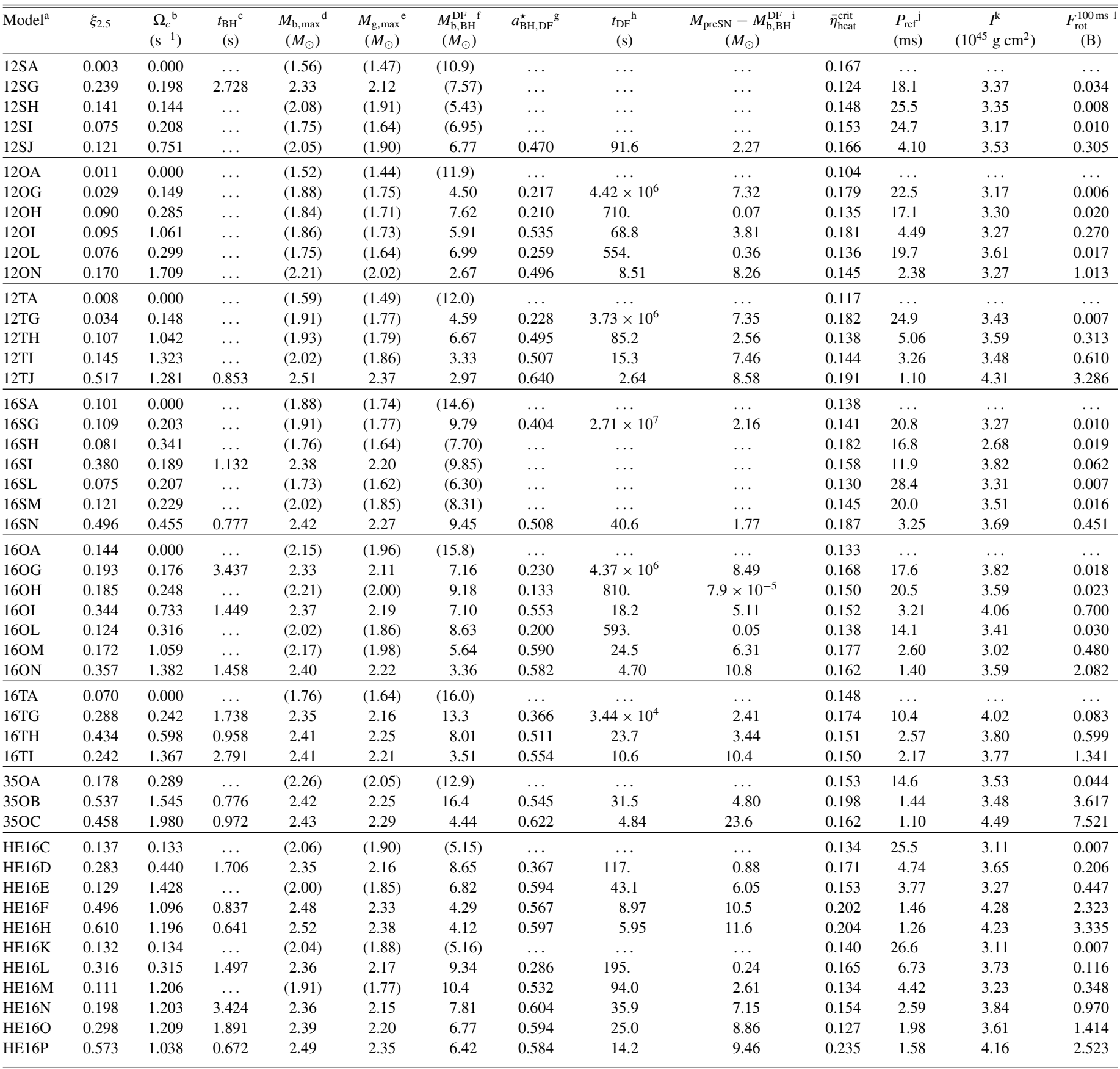

Notes.

${ }^{a}$ Model designation from Woosley \& Heger (2006). See the text for details.

${ }^{\mathrm{b}}$ Initial central angular velocity.

${ }^{c}$ Time elapsed between bounce and black hole formation. $\cdots$ indicates that no black hole formed within $3.5 \mathrm{~s}$ of bounce

${ }^{\mathrm{d}}$ Baryonic mass of the proto-neutron star at the time of black hole formation. If no black hole forms in $3.5 \mathrm{~s}$, we give the proto-neutron star baryonic mass at $3.5 \mathrm{~s}$.

e Gravitational mass of the proto-neutron star at the time of black hole formation. If no black hole forms in $3.5 \mathrm{~s}$, we give the proto-neutron star gravitational mass at $3.5 \mathrm{~s}$.

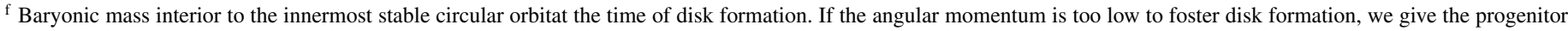
mass in parentheses instead.

g Dimensionless spin of the black hole when the disk forms. $\cdots$ indicates no disk forms.

h Twice the free-fall time of the mass element at the innermost stable circular orbit. $\cdots$ indicates no disk forms.

${ }^{\mathrm{i}}$ Baryonic mass outside of the black hole at disk formation. $\cdots$ indicates no disk forms.

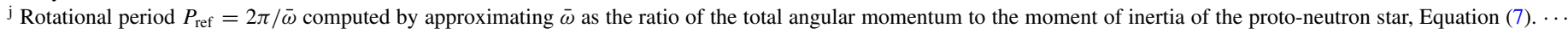
indicates a non-rotating model.

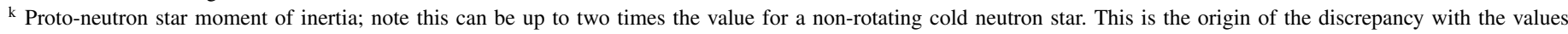

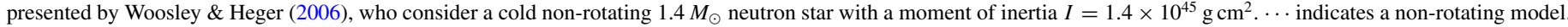

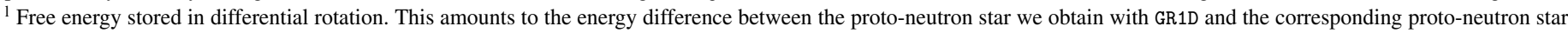
with the same total angular momentum and moment of inertia but assuming solid-body rotation. $\cdots$ indicates a non-rotating model. 


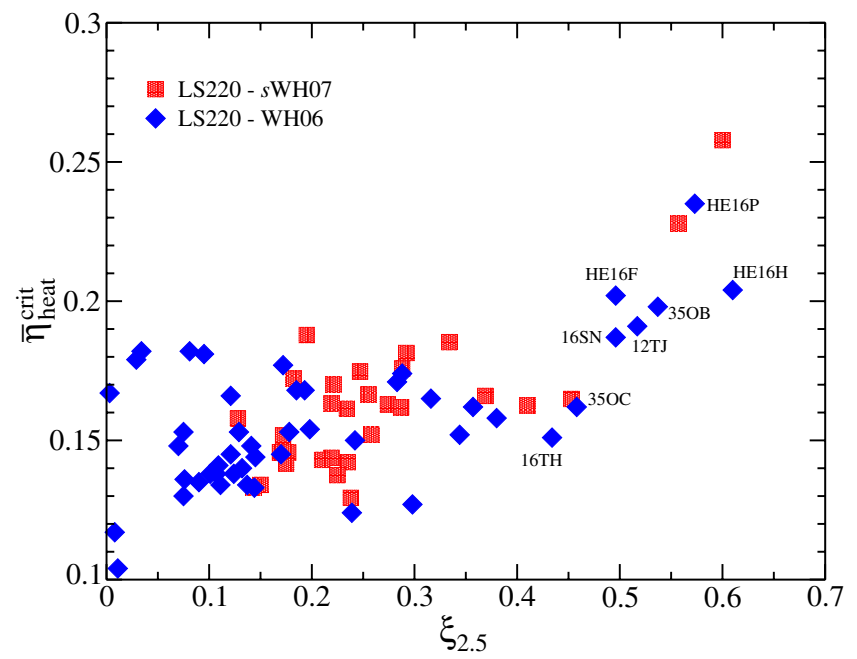

Figure 2. Illustration of the critical heating efficiency $\bar{\eta}_{\text {heat }}^{\text {crit }}$ vs. bounce compactness $\xi_{2.5}$ for our GR1D simulations of the Woosley \& Heger (2006) models, whose properties are summarized in Table 1 (blue diamonds). For comparison, we overplot the same quantity for the standard non-rotating core-collapse SN progenitor models of Woosley \& Heger (2007) evolved at solar metallicity (red squares). For the most part, the two distributions overlap, suggesting that the propensity to black hole formation and explosion is comparable for both. Only models with the fastest rotation rates achieve a larger compactness in excess of 0.4-0.5, but these may then be diverted from black hole formation through an early magneto-rotational explosion.

(A color version of this figure is available in the online journal.)

where we take $M=2.5 M_{\odot} . R\left(M_{\text {bary }}=2.5 M_{\odot}\right)$ is the radial coordinate that encloses $2.5 M_{\odot}$ of baryonic material at the time of core bounce (O'Connor \& Ott 2011).

Our simulations first demonstrate that most of the models have a small core compactness $\xi_{2.5}$. O'Connor \& Ott (2011) argue that a compactness of 0.45 represents a threshold value, for the neutrino mechanism, since above it an unrealistic neutrino-heating efficiency is required to prevent black hole formation. We further confirm this by determining the critical heating efficiency for the models in Table 1 via the same procedure as in O'Connor \& Ott (2011), to which we refer the reader for full details. We note that this criterion is for explosions via the neutrino mechanism and therefore neglects any magneto-rotational contribution to the powering of an explosion (Dessart et al. 2008), so this threshold value is probably a lower limit. We also stress that this criterion is based on spherically symmetric simulations with an efficient, but crude, approximation to neutrino transport. Therefore we suggest caution when attempting to interpret the outcome of a model based solely on the bounce compactness, since the adopted threshold value for black hole formation is a semiquantitative estimate based on where the slope of the required heating efficiency begins to clearly increase (see the discussion in O'Connor \& Ott 2011). When plotting the critical heating efficiency of the non-rotating solar metallicity stars from Woosley \& Heger (2007) determined in O'Connor \& Ott (2011), together with the generally fast-rotating progenitors of Woosley \& Heger (2006), we find that both data sets in fact overlap for the most part (Figure 2). In other words, in terms of compactness, most of these progenitors are similar to garden-variety, low-mass, non-rotating, progenitors and do not seem to have any more reason to form a black hole than, e.g., the red supergiant (RSG) star progenitors expected to produce SNe II-Plateau. As shown in Table 1, provided no explosion is launched, half of these models have not formed a black hole after $3.5 \mathrm{~s}$, and only $\sim 15 \%$ do within $\sim 1 \mathrm{~s}$. We note that this result is not so surprising given the small iron-core mass $\left(\sim 1.4 M_{\odot}\right)$ of most Woosley \& Heger (2006) models (see their Tables 1 and 2). The first conclusion from our work is therefore that most of the models presented here are rather unlikely to form a black hole and thus may fail in a very fundamental way to produce a collapsar, irrespective of their angular-momentum budget.

Within each sequence presented in Table 1, the models that form a black hole within $3.5 \mathrm{~s}$ of core bounce, and thus at least in principle susceptible to form a collapsar, are the faster rotating ones characterized by very weak mass-loss rates. These properties conspire to produce larger $\mathrm{CO}$ cores, more typical of more massive stars that do not rotate. In the following discussion, we group these models into several categories.

The first category are models which obviously do not give rise to an LGRB, either by the standard Type-I collapsar or the protomagnetar mechanism, because they contain too little angular momentum. Optimistically assuming a failed core-collapse SN, which is unlikely given the modest values of $\xi_{2.5}$, models $12 \mathrm{SG}$ $\left(\xi_{2.5}=0.239\right), 16 \mathrm{OG}\left(\xi_{2.5}=0.193\right), 16 \mathrm{SI}\left(\xi_{2.5}=0.380\right)$, and 16TG $\left(\xi_{2.5}=0.288\right)$ possess too little angular momentum in the remainder of the star to form a disk about the central black hole within $10^{6} \mathrm{~s}$ of collapse. This behavior is reflected by the stellar type at the time of death, i.e., a blue supergiant star for model 12 SG and an RSG star for models $16 \mathrm{OG}$ and 16TG; only 16SI is a W-R star at the time of death. Quantitatively, this can be further inspected in Table 1 where we include the disk formation time, the black hole mass and spin at that time, and the mass exterior to the disk. We define disk formation to be when the accreting material will first be supported at the innermost stable circular orbit about a black hole with the enclosed mass and angular momentum using the formulae of Bardeen et al. (1972). We estimate the disk formation time as twice the free-fall time of the innermost mass element that reaches a Keplerian velocity (O’Connor \& Ott 2011; Burrows 1986).

$$
t_{\mathrm{DF}}=2 \times \pi \sqrt{\frac{\left[r_{\mathrm{pre}-\mathrm{SN}}\left(M_{\mathrm{disk}}\right)\right]^{3}}{8 G M_{\mathrm{disk}}}},
$$

where $r_{\text {pre-SN }}\left(M_{\text {disk }}\right)$ is the radius of the disk-forming Lagrangian mass element in the pre-SN model. If no such mass element exists, no disk will form. In this case we include, instead of the enclosed black hole mass, the total pre-SN stellar mass in parentheses. In the four models mentioned above, either no disk forms or the disk formation time is $\gtrsim 10^{6} \mathrm{~s}$.

Additionally, we can discuss the potential for these models to form an LGRB via the proto-magnetar model. Using Equations (4)-(7), we calculate the free energy available in differential rotation at $100 \mathrm{~ms}$ after bounce. We also calculate a reference spin period $\left(P_{\text {ref }}=2 \pi / \bar{\omega}\right)$, measured at the onset of the neutrino-driven explosion, by assuming solid-body rotation for the entire proto-neutron star with the same total angular momentum and moment of inertia. The corresponding values are given in Table 1. In Figure 3, we show the free energy available in differential rotation at $100 \mathrm{~ms}$ and the spin period of the proto-neutron star at the onset of explosion. The total rotational energy of the proto-neutron star, estimated as $I \bar{\omega}^{2} / 2$, will increase as the proto-neutron star cools and contracts. In models 12SG, 16OG, 16SI, and 16TG, which are contained within the green (lightest shade) box of Figure $3, \lesssim 0.1 \mathrm{~B}$ of free energy could be extracted from differential rotation via the MRI and converted to explosion energy, much less than is needed for a magneto-rotational explosion. Also, the proto-neutron star spin 


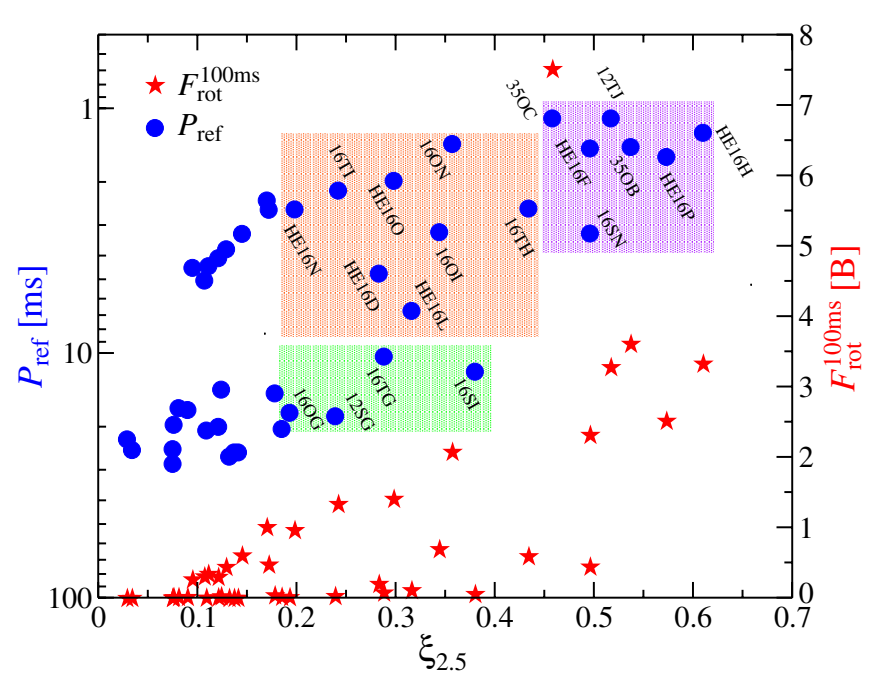

Figure 3. Reference proto-neutron star spin period, $P_{\text {ref }}$, taken at the onset of explosion (left axis, blue dots; Equation (7)) and the free energy stored in differential rotation $100 \mathrm{~ms}$ after bounce $F_{\text {rot }}^{100 \mathrm{~ms}}$ (right axis, red stars; Equation (4)) vs. bounce compactness $\xi_{2.5}$ for all rotating models in Table 1. While models with a low bounce compactness show a diversity in core-rotation properties, those with a high bounce compactness systematically have short spin periods and a large budget of free energy stored in the differential rotation. Shaded boxes refer to specific groupings of models discussed in the text. Using $\xi_{2.5}>0.45$ as a black hole formation criterion for non-rotating progenitors, we can qualitatively compare the reference spin periods of this figure to Metzger et al. (2011), who sketches the outcome of collapse as a function of progenitor spin and mass. From this, one would predict that none of the LGRB progenitor models studied here formed black holes.

(A color version of this figure is available in the online journal.)

periods are $\gtrsim 10 \mathrm{~ms},{ }^{7}$ significantly larger than the $\lesssim 2 \mathrm{~ms}$ periods required for the proto-magnetar model to reproduce classical LGRB energies (Metzger et al. 2011).

The second category are models with a larger angularmomentum budget but unfavorable bounce compactness. Although compact enough to lead to black hole formation within $3.5 \mathrm{~s}$ of core bounce, we find that the predicted critical heating efficiencies are similar to that expected for a standard $15 M_{\odot}$ nonrotating RSG progenitor star (O'Connor \& Ott 2011). These properties make them unlikely collapsar progenitors, but in contrast, make them ideal candidates for proto-magnetar formation, and perhaps LGRBs through that channel. These models include $16 \mathrm{OI}\left(\xi_{2.5}=0.344\right), 16 \mathrm{ON}\left(\xi_{2.5}=0.357\right), 16 \mathrm{TH}$ $\left(\xi_{2.5}=0.434\right), 16 \mathrm{TI}\left(\xi_{2.5}=0.242\right), \operatorname{HE} 16 \mathrm{D}\left(\xi_{2.5}=0.283\right)$, HE16L $\left(\xi_{2.5}=0.316\right)$, HE16N $\left(\xi_{2.5}=0.198\right)$, and HE16O $\left(\xi_{2.5}=0.298\right)$, and are contained in the orange (medium shade) box of Figure 3. In addition to having critical heating efficiencies similar to what is needed to explode typical low-mass massive stars, the free energy available in rotation is $\mathcal{O}(1 \mathrm{~B})$. This energy may be converted to explosion energy via the magneto-rotational mechanism. The spin period of these proto-neutron stars is in the range 1-6 ms, thus on the order of what is needed for the proto-magnetar model of LGRBs (Metzger et al. 2011).

Eventually, the fastest rotating progenitor models evolved with a strongly inhibited stellar-wind mass loss represent more suitable collapsar candidates, although each model has caveats. This set is contained in the purple (darkest shade) box of Figure 3 and includes models $12 \mathrm{TJ}\left(\xi_{2.5}=0.517\right), 16 \mathrm{SN}\left(\xi_{2.5}=0.496\right)$,

\footnotetext{
7 Even taking into account the spin up due to the PNS cooling and contraction, which will decrease the moment of inertia from the value in Table 1 to $\sim 0.4 M_{\mathrm{PNS}} R_{\mathrm{PNS}}^{2} \sim 1.6 \times 10^{45}\left(M / 1.4 M_{\odot}\right)(R / 12 \mathrm{~km})^{2}$ (Metzger et al. 2011), or roughly a factor of two, the spin periods are $\gtrsim 5 \mathrm{~ms}$.
}

35OB $\left(\xi_{2.5}=0.537\right)$, and 35OC $\left(\xi_{2.5}=0.458\right)$. Model $12 \mathrm{TJ}$ will form a $2.37 M_{\odot}$ (gravitational mass) black hole $0.85 \mathrm{~s}$ after core bounce, followed by a Keplerian disk after $2.64 \mathrm{~s}$, with a potential ejecta mass of $8.57 M_{\odot}$. However, much like the models in the previous category, model $12 \mathrm{TJ}$ has $\sim 3 \mathrm{~B}$ of free energy available in rotation that may lead to a magneto-rotational explosion early-on, preventing collapsar formation. This model is evolved at $1 \%$ solar metallicity, with an additional mass-loss rate scaling of 0.1 , equivalent to an overall evolution at $10^{-4}$ solar metallicity, much below that observed for LGRB/SN sites. We find that models HE16F, HE16H, and HE16P have similar characteristics to model 12TJ. Model $16 \mathrm{SN}$ forms a $2.27 M_{\odot}$ black hole $0.78 \mathrm{~s}$ after bounce. Being evolved at an effective metallicity of 0.01 solar, it has a lower angular-momentum budget at death and is thus more likely to avoid a magneto-rotational explosion. However, it forms a Keplerian disk only $40.6 \mathrm{~s}$ after core bounce, with only $1.77 M_{\odot}$ left over for the SN ejecta. Such characteristics might in fact be more amenable to reproduce recent observations of LGRB/SNe characterized by a very early and narrow light-curve peak, as witnessed for example for GRB100316D/SN 2010bh (Chornock et al. 2010). They may even explain why no $\mathrm{SN}$ is found in association with some nearby LGRBs (Fynbo et al. 2006). Finally, models 35OB and 35OC form a black hole within 0.78 and $0.97 \mathrm{~s}$ of bounce, respectively. Model $35 \mathrm{OB}$ will accrete $\sim 16.4 M_{\odot}$ before a Keplerian disk forms $\sim 31.5 \mathrm{~s}$ after the onset of collapse; $4.8 M_{\odot}$ is then available for the $\mathrm{SN}$ ejecta. With the $35 \mathrm{OC}$ model, a disk forms very quickly after collapse, in $4.8 \mathrm{~s}$, and a significant amount of mass is exterior to the disk, $23.6 M_{\odot}$, and thus much too large to accommodate inferred LGRB/SN ejecta masses. However, the propensity to collapsar formation of the $35 \mathrm{OB}$ and 35OC models may be ill-founded if the MRI is successful at powering a magneto-rotational explosion. The free energy available in rotation is huge, i.e., on the order of 4-7.5 B. In fact, in the 2D magneto-hydrodynamic simulations of Dessart et al. (2008) based on the 35OC model, it was found that, despite the large progenitor compactness, a magneto-rotational explosion was initiated $\sim 200 \mathrm{~ms}$ after core bounce and that the proto-neutron star mass decreased thereafter, never reaching the mass threshold for black hole formation. In our models, the proto-neutron stars in models $35 \mathrm{OB}$ and $35 \mathrm{OC}$ have $\sim 30-70$ B of total rotational energy at the onset of explosion, amply matching the inferred energies of observed hypernovae. These inferences are based on the assumption that energy extraction from the differentially rotating layers at the proto-neutron star surface is efficient and can power an explosion. Failing to do so, black hole formation would result, although the question of energy extraction from the disk for the powering of a GRB would then arise. Detailed multi-dimensional core-collapse simulations need to be carried out to investigate the efficiencies of magnetic/rotational/hydrodynamical instabilities for the transport of angular momentum and the extraction of rotational energy.

\section{DISCUSSION}

In this paper, we have performed 1D general-relativistic hydrodynamical simulations with GR1D of the collapse, bounce, and post-bounce phases of the LGRB candidates of Woosley \& Heger (2006) to investigate their propensity to black hole and disk formation. We find that these progenitors are at odds with the proposed criteria for a collapsar progenitor or with the inferred properties of observed LGRB/SNe, namely, an Hdeficient He-poor W-R star with a massive iron core (equivalent 
to a large compactness), a large angular momentum to form a Keplerian disk soon after black hole formation, an ejecta of $\sim 10 M_{\odot}$, and an evolution at about 0.1 solar metallicity. A critical aspect that we focus on in this study is the compactness of the progenitor cores at bounce, a quantity that helps diagnose the likelihood of black hole formation.

We group the Woosley \& Heger (2006) models in different categories according to their suitability for producing collapsars.

1. Models with a dimensionless Kerr spin parameter greater than unity at an enclosed mass of $3 M_{\odot}$, i.e., the models identified by Woosley \& Heger (2006) as having the best potential for collapsar formation, fail to collapse when evolved with GR1D. Their cores are spinning so fast that the associated centrifugal acceleration leads them into expansion. All these models are evolved until death in KEPLER without magnetic fields and centrifugal forces, which seems questionable given the unrealistically short spin periods at collapse.

2. Models evolved with magnetic fields produce much lower rotation rates and most collapse with GR1D.

3. Of those evolved with magnetic fields, models with moderate rotation produce progenitors with a small compactness comparable to that characterizing the low-mass massivestar models proposed as progenitors of garden-variety corecollapse SNe. A small fraction of these is endowed with sufficient angular momentum to make a proto-magnetar, and thus a potential channel for producing LGRBs.

4. A few models (12TJ, 16SN) with the fastest rotation possess a large compactness favorable for black hole formation and sufficient angular momentum for the formation of a Keplerian disk, but they require evolution at metallicities in the range $0.0001-0.01 Z_{\text {sol }}$, significantly lower than the metallicity of a few tenths solar or even higher at which these LGRB/SNe are found (Modjaz et al. 2008; Levesque et al. 2010a, 2010b). Although in many respects very attractive for forming a collapsar (if we ignore its huge core angular momentum), model $350 \mathrm{O}$ is characterized by a large envelope mass of $\sim 23 M_{\odot}$, which is a factor of 2-10 times larger than the inferred ejecta mass of LGRB/SNe discovered so far (for a summary, see Berger et al. 2011). We note that these models have a large angular momentum in the core, like models in the previous category, and may thus experience a magneto-rotational explosion preventing collapsar formation.

Our quantitative study thus spells out the various shortcomings of these progenitor stars for producing collapsars. Even in those models that have the right compactness for black hole formation and sufficient angular momentum for disk formation, it is still unresolved today how they would avoid the magnetorotational mechanism of explosion that is used to explain hypernovae (LeBlanc \& Wilson 1970; Bisnovatyi-Kogan et al. 1976; Wheeler et al. 2000; Yamada \& Sawai 2004; Moiseenko et al. 2006; Burrows et al. 2007; Dessart et al. 2008; Takiwaki \& Kotake 2011). The difficulty of forming a black hole and avoiding a magneto-rotational explosion in fast-rotating cores, at least in the models of Woosley \& Heger (2006), lends credence to the proto-magnetar model of LGRB/SNe.

Uncertainties in mass loss at low metallicity, and in particular during transient phases of dynamical mass loss as observed in some luminous blue variable stars, is an issue, since it may completely dominate the mass lost in the form of a weaker, but secular, steady-state wind (Owocki et al. 2004). This uncertain mass-loss rate plagues more severely the evolution of highermass stars, since $15-20 M_{\odot}$ stars stay farther away from the Eddington limit, and overall lose little mass, even at solar metallicity. By what mechanism, at what rate, and during what phases a $100 M_{\odot}$ star loses mass (and angular momentum) is much less well known and this directly conditions the final mass and iron-core mass at collapse.

Overall, this suggests that studies of collapsar progenitors would benefit from a second look. Angular momentum is key in the current collapsar and proto-magnetar models, but there is a stiff requirement on the progenitor compactness to speculate on its propensity for forming a black hole, and thus for producing an LGRB through one or the other channel. A major step forward in resolving those issues would be to conduct massivestar evolution with rotation, centrifugal force, and magnetic fields always all the way to the formation of a degenerate neutronized core on the verge of collapse. This would allow a straightforward comparison of results between groups, and an easy determination of the compactness using GR1D to test the suitability of the core for black hole formation.

The ultimate check on the collapsar model requires multidimensional simulations covering the whole evolution from progenitor collapse, bounce, failed explosion during the protoneutron star phase, formation of a black hole followed by the formation of a Keplerian disk, and the powering of a $\sim 10 \mathrm{~B}$ $\mathrm{SN}$ ejecta. As we emphasize, black hole formation is perhaps one of the most difficult steps in this sequence of events, and in that respect, renders the proto-magnetar channel quite attractive for the production of hypernovae and LGRBs. The diversity of LGRB/SNe, the existence of SN-less LGRBs and of LGRBless hypernovae, may in fact call for a variety of formation channels for these rare events, including both collapsars and proto-magnetars.

We acknowledge fruitful discussions with R. Hirschi, A. Beloborodov, and T. Piro. We also thank Stan Woosley for his comments on a draft version of this paper. This research is supported in part by the National Science Foundation under grant Nos. AST-0855535 and OCI-0905046 and by the Sherman Fairchild Foundation. E.O. is supported in part by a postgraduate fellowship from the Natural Sciences and Engineering Research Council of Canada (NSERC). The computations were performed at Caltech's Center for Advanced Computing Research on the cluster "Zwicky" funded through NSF grant No. PHY-0960291 and the Sherman Fairchild Foundation.

\section{REFERENCES}

Akiyama, S., Wheeler, J. C., Meier, D. L., \& Lichtenstadt, I. 2003, ApJ, 584, 954

Aloy, M. A., Müller, E., Ibáñez, J. M., Martí, J. M., \& MacFadyen, A. 2000, ApJ, 531, L119

Balbus, S. A., \& Hawley, J. F. 1991, ApJ, 376, 214

Bardeen, J. M., Press, W. H., \& Teukolsky, S. A. 1972, ApJ, 178, 347

Berger, E., Chornock, R., Holmes, T. R., et al. 2011, ApJ, 743, 204

Bethe, H. A. 1990, Rev. Mod. Phys., 62, 801

Bethe, H. A., \& Wilson, J. R. 1985, ApJ, 295, 14

Bisnovatyi-Kogan, G. S., Popov, I. P., \& Samokhin, A. A. 1976, Ap\&SS, 41, 287

Bucciantini, N., Quataert, E., Arons, J., Metzger, B. D., \& Thompson, T. A. 2007, MNRAS, 380, 1541

Bucciantini, N., Quataert, E., Arons, J., Metzger, B. D., \& Thompson, T. A. 2008, MNRAS, 383, L25

Bucciantini, N., Quataert, E., Metzger, B. D., et al. 2009, MNRAS, 396, 2038 Buras, R., Janka, H.-T., Rampp, M., \& Kifonidis, K. 2006a, A\&A, 457, 281 Buras, R., Rampp, M., Janka, H.-T., \& Kifonidis, K. 2006b, A\&A, 447, 1049 Burrows, A. 1986, ApJ, 300, 488 
Burrows, A., Dessart, L., Livne, E., Ott, C. D., \& Murphy, J. 2007, ApJ, 664 416

Burrows, A., Hayes, J., \& Fryxell, B. A. 1995, ApJ, 450, 830

Burrows, A., \& Lattimer, J. M. 1983, ApJ, 270, 735

Burrows, A., Livne, E., Dessart, L., Ott, C. D., \& Murphy, J. 2006, ApJ, 640, 878

Cantiello, M., Yoon, S., Langer, N., \& Livio, M. 2007, A\&A, 465, L29

Chornock, R., Berger, E., Levesque, E. M., et al. 2010, arXiv:1004.2262

Demorest, P. B., Pennucci, T., Ransom, S. M., Roberts, M. S. E., \& Hessels, J. W. T. 2010, Nature, 467, 1081

Dessart, L., Burrows, A., Livne, E., \& Ott, C. D. 2008, ApJ, 673, L43

Dessart, L., \& Hillier, D. J. 2008, MNRAS, 383, 57

Dessart, L., Hillier, D. J., Li, C., \& Woosley, S. 2012, MNRAS, in press (arXiv:1205.5349)

Dimmelmeier, H., Ott, C. D., Marek, A., \& Janka, H.-T. 2008, Phys. Rev. D, 78,064056

Fischer, T., Whitehouse, S. C., Mezzacappa, A., Thielemann, F.-K., \& Liebendörfer, M. 2009, A\&A, 499, 1

Fynbo, J. P. U., Watson, D., Thöne, C. C., et al. 2006, Nature, 444, 1047

Georgy, C., Meynet, G., Walder, R., Folini, D., \& Maeder, A. 2009, A\&A, 502, 611

Goldreich, P., \& Weber, S. V. 1980, ApJ, 238, 991

Guetta, D., \& Della Valle, M. 2007, ApJ, 657, L73

Hanke, F., Marek, A., Müller, B., \& Janka, H.-T. 2011, ApJ, submitted (arXiv:1108.4355)

Hebeler, K., Lattimer, J. M., Pethick, C. J., \& Schwenk, A. 2010, Phys. Rev. Lett., 105, 161102

Heger, A., Langer, N., \& Woosley, S. E. 2000, ApJ, 528, 368

Heger, A., Woosley, S. E., \& Spruit, H. C. 2005, ApJ, 626, 350

Herant, M., Benz, W., Hix, W. R., Fryer, C. L., \& Colgate, S. A. 1994, ApJ, 435,339

Hirschi, R., Meynet, G., \& Maeder, A. 2004, A\&A, 425, 649

Hirschi, R., Meynet, G., \& Maeder, A. 2005, A\&A, 443, 581

Iwamoto, K., Mazzali, P. A., Nomoto, K., et al. 1998, Nature, 395, 672

Janka, H.-T., \& Müller, E. 1996, A\&A, 306, 167

Kitaura, F. S., Janka, H.-T., \& Hillebrandt, W. 2006, A\&A, 450, 345

Komissarov, S. S., \& Barkov, M. V. 2007, MNRAS, 382, 1029

Lattimer, J. M., \& Swesty, F. D. 1991, Nucl. Phys. A, 535, 331

LeBlanc, J. M., \& Wilson, J. R. 1970, ApJ, 161, 541

Levesque, E. M., Kewley, L. J., Berger, E., \& Zahid, H. J. 2010a, AJ, 140, 1557

Levesque, E. M., Soderberg, A. M., Foley, R. J., et al. 2010b, ApJ, 709, L26

Liebendörfer, M., Messer, O. E. B., Mezzacappa, A., et al. 2004, ApJS, 150, 263

Limongi, M., \& Chieffi, A. 2006, ApJ, 647, 483

Lindner, C. C., Milosavljević, M., Couch, S. M., \& Kumar, P. 2010, ApJ, 713, 800

MacFadyen, A. I., \& Woosley, S. E. 1999, ApJ, 524, 262

MacFadyen, A. I., Woosley, S. E., \& Heger, A. 2001, ApJ, 550, 410
Maeder, A., \& Meynet, G. 2000, ARA\&A, 38, 143

Mazzali, P. A., Deng, J., Maeda, K., et al. 2002, ApJ, 572, L61

Metzger, B. D. 2010, in ASP Conf. Ser. 432, New Horizons in Astronomy: Frank N. Bash Symposium 2009, ed. L. M. Stanford, J. D. Green, L. Hao, \& Y. Mao (San Francisco, CA: ASP), 81

Metzger, B. D., Giannios, D., Thompson, T. A., Bucciantini, N., \& Quataert, E. 2011, MNRAS, 413, 2031

Meynet, G., \& Maeder, A. 2000, A\&A, 361, 101

Meynet, G., \& Maeder, A. 2005, A\&A, 429, 581

Milosavljević, M., Lindner, C. C., Shen, R., \& Kumar, P. 2012, ApJ, 744, 103

Modjaz, M., Kewley, L., Kirshner, R. P., et al. 2008, AJ, 135, 1136

Moiseenko, S. G., Bisnovatyi-Kogan, G. S., \& Ardeljan, N. V. 2006, MNRAS, 370,501

Murphy, J. W., \& Burrows, A. 2008, ApJ, 688, 1159

Nordhaus, J., Burrows, A., Almgren, A., \& Bell, J. 2010, ApJ, 720, 694

Obergaulinger, M., Cerdá-Durán, P., Müller, E., \& Aloy, M. A. 2009, A\&A, 498, 241

O’Connor, E., \& Ott, C. D. 2010, Class. Quantum Grav., 27, 114103

O'Connor, E., \& Ott, C. D. 2011, ApJ, 730, 70

Ott, C. D., Burrows, A., Thompson, T. A., Livne, E., \& Walder, R. 2006, ApJS, 164,130

Ott, C. D., Reisswig, C., Schnetter, E., et al. 2011, Phys. Rev. Lett., 106, 161103

Owocki, S. P., Gayley, K. G., \& Shaviv, N. J. 2004, ApJ, 616, 525

Özel, F., Baym, G., \& Güver, T. 2010, Phys. Rev. D, 82, 101301

Pastorello, A., Zampieri, L., Turatto, M., et al. 2004, MNRAS, 347, 74

Pejcha, O., \& Thompson, T. A. 2012, ApJ, 746, 106

Petrovic, J., Langer, N., Yoon, S.-C., \& Heger, A. 2005, A\&A, 435, 247

Proga, D., MacFadyen, A. I., Armitage, P. J., \& Begelman, M. C. 2003, ApJ, 599, L5

Sekiguchi, Y., \& Shibata, M. 2011, ApJ, 737, 6

Spruit, H. C. 2002, A\&A, 381, 923

Steiner, A. W., Lattimer, J. M., \& Brown, E. F. 2010, ApJ, 722, 33

Sumiyoshi, K., Yamada, S., \& Suzuki, H. 2007, ApJ, 667, 382

Takiwaki, T., \& Kotake, K. 2011, ApJ, 743, 30

Thompson, T. A., Quataert, E., \& Burrows, A. 2005, ApJ, 620, 861

Wellstein, S., \& Langer, N. 1999, A\&A, 350, 148

Wheeler, J. C., Yi, I., Höflich, P., \& Wang, L. 2000, ApJ, 537, 810

Woosley, S. E. 1993, ApJ, 405, 273

Woosley, S. E. 2011, arXiv:1105.4193

Woosley, S. E., Eastman, R. G., \& Schmidt, B. P. 1999, ApJ, 516, 788

Woosley, S. E., \& Heger, A. 2006, ApJ, 637, 914

Woosley, S. E., \& Heger, A. 2007, Phys. Rep., 442, 269

Yamada, S., \& Sawai, H. 2004, ApJ, 608, 907

Yoon, S.-C., \& Langer, N. 2005, A\&A, 443, 643

Yoon, S.-C., Langer, N., \& Norman, C. 2006, A\&A, 460, 199

Zahn, J.-P., Brun, A. S., \& Mathis, S. 2007, A\&A, 474, 145

Zhang, W., Woosley, S. E., \& Heger, A. 2004, ApJ, 608, 365 